\title{
On the interpretation of the Nernst effect measurements in the cuprates
}

\author{
Iddo Ussishkin ${ }^{1}$ and S. L. Sondhi ${ }^{2}$ \\ ${ }^{1}$ William I. Fine Theoretical Physics Institute, University of Minnesota, Minneapolis, Minnesota 55455 \\ ${ }^{2}$ Department of Physics, Princeton University, Princeton, New Jersey 08544
}

\begin{abstract}
We consider the large Nernst signal discovered by Ong and collaborators in hole-doped cuprates, in particular in the pseudogap regime. Based on our previous quantitative calculations together with Huse [Phys. Rev. Lett. 89, 287001 (2002)], we discuss the interpretation of the experimental observations as arising from superconducting fluctuations and its relation to the vortex scenario proposed by Ong. We also comment on the implications of the Nernst analysis for understanding the full range of pseudogap phenomena.
\end{abstract}

The Nernst effect is the appearance of a transverse electric field $E_{y}$ in response to a temperature gradient $(-\nabla T) \| \hat{\mathbf{x}}$, in the presence of a perpendicular magnetic field $\mathbf{B} \| \hat{\mathbf{z}}$ and under open circuit conditions (Fig. 1). Conventional wisdom holds that the effect is small in the normal state of metals where transport by quasiparticles is dominant, as is indeed observed, e.g., in the normal state of conventional superconductors. In contrast, conventional wisdom also holds that the Nernst effect is large in the superconducting state where a new set of excitations - vortices - now enter the picture.

The intuition behind the wisdom is easily stated. The temperature gradient requires that a heat current flow across the sample in the $x$ directions while the open circuit requires that there be no associated electrical current. With quasiparticles this is accomplished by counterflows of hot and cold electrons which then give rise to opposed Hall voltages in the presence of the magnetic field and nearly cancel. Once vortices are available, they can carry heat without transporting charge and by the Josephson relation for phase slips they support a voltage transverse to their direction of flow. This intuition is not perfect - e.g., in particle-hole symmetric situations the two species of quasiparticles can carry a heat current without transporting charge and thus give Nernst signals that add, while there isn't really a theory of the Nernst effect from vortex motion as we explain below. Nevertheless, the expected contrast works fairly well in practice.

Recent measurements of the Nernst effect in the high-temperature cuprate superconductors by Ong and collaborators ${ }^{1.2}$ have challenged this conventional wis-

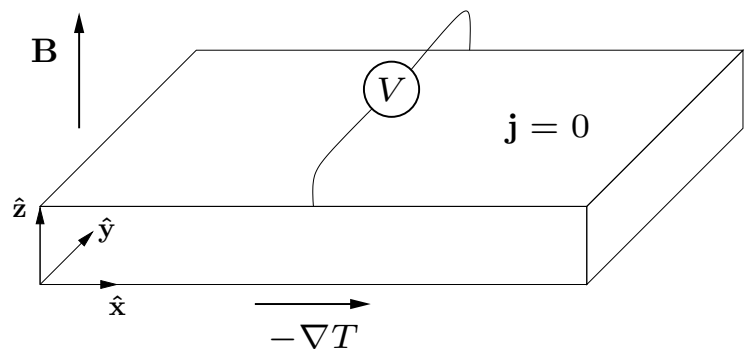

FIG. 1: Basic geometry of the Nernst experiment. dom and pose a problem for the theory of the Nernst effect as well as for our evolving understanding of the cuprates. More precisely, the experiments have uncovered a a large Nernst signal in the non-superconducting state of hole-doped cuprates, at temperatures well above the critical temperature $T_{c}$. The effect is particularly pronounced in underdoped samples, extending well into the "pseudogap" region of the cuprate phase diagram. We note that experimentally its existence was observed

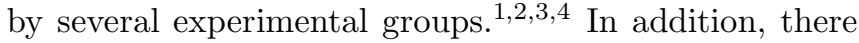
are several earlier precursor observations reported in optimally doped samples $\frac{5,6,7,8,9}{4}$

At issue then, is how to account for a large Nernst signal in the "normal" state of the system. The answer is presumably related to the fact that the normal state in the cuprates is anything but normal over much of the phase diagram - hence our use of quotation marks. Indeed, much theoretical and experimental effort has been directed at trying to understand the physics of the non-superconducting pseudogap region. In this region, anomalous behavior is observed in a variety of properties roughly below a crossover temperature scale $T^{*}$, including a pseudogap in the spectrum which lends the region its name $\frac{10}{0}$ The Nernst effect thus presents a new challenge in this context.

The simplest proposal that can account for the Nernst data uses no physics beyond the conventional wisdom. As the effect is large in the presence of superconductivity and small in its absence one is led to the conjecture that the pseudogap region contains significant superconducting fluctuations that give rise to the observed Nernst signal. At this level of generality, this is the interpretation originally put forward by Ong and collaborators $\frac{1.2}{.1}$ It is also in accord with an influential set of ideas on the pseudogap that attribute its various anomalies to fluctuating superconductivity 11.12 .13

More specifically, Ong and collaborators have argued that the strong Nernst signal results from vortex degrees of freedom which arise when the amplitude of the order parameter is essentially fixed, while the phase is allowed to fluctuate. In this picture the onset temperature of the Nernst effect as observed in experiment, $T_{\text {onset }}$, is taken to be as a measure of the onset of the phase fluctuation regime. While this picture is intuitively appealing, it has not yet proven possible to carry out computations based 
on it. In contrast, we and Huse have recently performed a calculation of the contribution of Gaussian fluctuations to the Nernst signal. ${ }^{17}$ In our work, we found that in overdoped samples of $\mathrm{La}_{2-x} \mathrm{Sr}_{x} \mathrm{CuO}_{4}$ (LSCO), the data well above $T_{c}$ quantitatively agrees with Gaussian fluctuations with a reasonable choice of a coherence length $\xi$ and simply setting the mean field critical temperature $T_{c}^{\mathrm{MF}}$, which enters all Gaussian computations, equal to $T_{c}$. In underdoped samples, the result for Gaussian fluctuations is smaller than the observed signal if we keep $T_{c}^{\mathrm{MF}} \approx T_{c}$ (and use the same $\xi$ ). It was argued that a qualitative understanding may be achieved by assuming that $T_{c}^{\mathrm{MF}}$ is higher than the actual $T_{c}$ in the underdoped regime due to strong fluctuations. Altogether we believe that this work provides strong support to the interpretation of the Nernst experiments as the work of superconducting fluctuations. As the previous discussion was rather compressed and there are several delicate points, in this article we will provide a fuller discussion of the issues involved. Specifically, we will (1) clarify some aspects of our analysis, (2) discuss its relation to the vortex interpretation, and (3) attempt to bound what we feel the Nernst effect is telling us regarding the physics of the pseudogap. We note that the Nernst effect was also considered by several other groups along similar or related lines. 18.19 .20 .21

Before proceeding, we should note that there is still considerable debate on the physics of the pseudogap region. While it is beyond our brief here to attempt a survey of all the work on this problem, we note that the ideas around range from RVB (resonating valence bond) proposals built on spin-charge separation, $\frac{14.22}{24}$ through fluctuating charge and spin order ideas, 23.24 to the DDW ( $d$ density wave) proposal in which the pseudogap is a phase with a distinct broken symmetry ${ }^{25}$ However there is not, at present, a viable alternative scenario for the Nernst experiments based on any of these proposals which is distinct from invoking superconducting fluctuations. For example, recently Oganesyan and one of us have studied the contribution of quasiparticles to the Nernst signal, in particular in connection with the DDW scenario for the pseudogap region and failed to find a significant effect ${ }^{26}$ This computation is of interest as similar computations have supported the plausibility of the DDW idea in accounting for the photoemission experiments in the pseudogap region.

We now turn to a telegraphic discussion of our main points.

1. Order parameter dynamics: We assume that the dynamics of the superconducting order parameter in the regime of interest is classical. This is likely a safe assumption above and near $T_{c}$ provided the system is not too underdoped. This allows us to write a stochastic timedependent Ginzburg-Landau equation for the evolution of the order parameter $\psi$,

$$
\partial_{t} \psi=-\Gamma \frac{\delta F}{\delta \psi}+\zeta
$$

where $F[\psi]=|\nabla \psi|^{2}+a|\psi|^{2}+b|\psi|^{4}+\ldots$ is the LandauGinzburg-Wilson functional ${ }^{27}$ This is Model A for critical dynamics in Hohenberg and Halperin's classification ${ }^{28}$ and assumes that the slowest dynamics is that of the order parameter.

It is important to note that this equation already contains in it all of the physics invoked by Emery and Kivelson, by Ong and collaborators and by ourselves. Close to $T_{c}$ there is a region where phase fluctuations dominate. In this region it is possible, at least in principle, to discuss the physics in terms of the vortex degrees of freedom. In $d=2$ this is rigorously so near the Kosterlitz-Thouless transition. In $d=3$ while there are discussions of the phase transition in terms of vortex loops ${ }^{29}$ these are much more complicated and not nearly as useful. As the temperature is increased, the density of the vortices increases and amplitude fluctuations become important in addition to the phase fluctuations $\frac{30}{2}$ As the temperature is increased further, another approximation becomes available, namely to neglect the quartic term and consider Gaussian fluctuations. The temperatures in this region are above $T_{c}^{\mathrm{MF}}$, the temperature at which the coefficient of $|\psi|^{2}$ in the free energy vanishes. Both amplitude and phase fluctuations are important in this region, and in a vortex picture these may be thought of as arising from a dense soup of vortices. [We note that this discussion holds for BCS superconductors as well. In this case, the difference between $T_{c}$ and $T_{c}^{\mathrm{MF}}$ is $\mathcal{O}\left(\Delta / E_{F}\right)$, as given by the Brout criterion. Critical behavior is observed in a much narrower region, given by the Ginzburg criterion as $\mathcal{O}\left(\Delta / E_{F}\right)^{4}$.]

So nothing fundamental is at issue between invoking vortices and our work - there is a single curve for the Nernst coefficient for which we have calculated the high temperature limit $\stackrel{31}{1}$ If we had failed to find a significant answer, it would have been hard to credit superconducting fluctuations with very much effect. Of course this is only one quantity - one will need a similar convergence between theory and experiment for some other to put the scenario on a really firm footing.

2. A few caveats: Before we continue, we should consider the limitations of our analysis. As already said, we assume classical fluctuations, and while this is always true asymptotically close to $T_{c}$, using it over a broad range of temperatures does require the dynamics to be more robustly classical.

In our treatment we consider the dynamics of the order parameter alone, and neglect all other degrees of freedom. Consequently quasiparticle contributions are ignored. Although this can be justified in BCS (including $d$-wave) superconductors, for cuprates the microscopic picture is not yet settled, and this assumption cannot be rigorously justified. We also neglect the possibility that other fields may acquire a slow dynamics. Here, the main candidate appears to be the energy field. Including it would lead to model $\mathrm{C}$ (but we note that models A and $\mathrm{C}$ yield the same result for the Nernst signal in the Gaussian approximation). In these systems, slow dynam- 
ics may also arise due to other types of incipient ordering (e.g., stripes, antiferromagnetic order) that may in principle also affect the Nernst signal.

As for the choice of dynamics for the order parameter, we make the conventional assumption that all parameters are temperature-independent, except for the coefficient of the quadratic term which varies as $T-T_{c}^{\mathrm{MF}}$. In reality, these coefficients may acquire a non-trivial temperature dependence, which depends on the underlying microscopics. We also assume particle-hole symmetry (or purely relaxational dynamics for the order parameter). Breaking of this symmetry may lead to a modification of our results.

Having stated these various caveats, we argue that nevertheless the analysis of the model A does provide important insight for the discussion of the Nernst experiments.

3. On the vortex "transport entropy": In the past, when describing the Nernst effect in terms of vortices on a phenomenological level, it has been customary to identify the "transport entropy" of a vortex, $S_{\phi} \underline{32}$ In the Nernst setup the temperature gradient then applies a force $\mathbf{f}=-S_{\varphi} \nabla T$ on the vortices. In this picture, the "transport entropy" also describes the heat carried by a vortex. Based on these definitions, the "transport entropy" of the vortex may be extracted from a Nernst measurement or calculation. However, although it has often been suggested in the literature that this quantity is related to the entropy of the vortex core, its relation to vortex properties remains to be clarified.

In this context, the calculation of Caroli and Maki ${ }^{33}$ for the "transport entropy" has often been cited in the literature. They considered the drift motion of the vortex lattice in the ordered state at the mean field level (see also Troy and Dorsey ${ }^{34}$ ). As we will discuss in detail elsewhere, the original calculation has to be corrected (so that the microscopic results for the heat current indeed match those of the TDGL near $T_{c}$ ) and modified to properly account for magnetization currents (see Ref. 35), and the correct result is rather surprising at first sight: The Nernst signal (and hence the "transport entropy") vanishes in this approach! This is in spite of the fact that there is a free energy cost associated with the vortex core in the model. At a hand-waving level, this result may be understood by noting that (1) the mean field equation is effectively at zero temperature, and (2) the solution involves an order parameter configuration drifting in its ground state and carrying no entropy of its own.

The bottom line of this discussion is that while the Nernst signal may be described in terms of vortices moving along the temperature gradient and creating electric field by phase slips, performing a calculation in this framework is non-trivial. In particular, the "transport entropy" is not obviously related to the properties of the vortex core, 36 and it is not clear if it has any useful meaning beyond its relation to the transport properties of the vortex state.

4. Gaussian results: Above $T_{c}^{\mathrm{MF}}$, the Gaussian approximation becomes available and is analytically tractable. We briefly describe the results of our calculation in this regime. We first note that theoretically we calculate the conductivity and thermoelectric tensors, $\sigma$ and $\alpha$, and not the Nernst effect directly. The Nernst signal is then related to these linear response coefficients by

$$
\frac{E_{y}}{(-\nabla T)_{x}}=\frac{\alpha_{x y} \sigma_{x x}-\alpha_{x x} \sigma_{x y}}{\sigma_{x x}^{2}+\sigma_{x y}^{2}} \approx \frac{\alpha_{x y}}{\sigma_{x x}} .
$$

The last expression is an approximation that can often be justified in the present context. We note that the behavior of the Nernst signal is dominated by the behavior of the transverse thermoelectric response $\alpha_{x y}$, making it the quantity of interest in our discussion. We also note that to observe an appreciable Nernst signal requires $\sigma_{x x}$ to be small. This is one of the main reasons the contribution of superconducting fluctuations to the Nernst signal is not observed in conventional low-temperature superconductors.

For the contribution of superconducting fluctuations to $\alpha_{x y}$ (above $T_{c}$ and to linear order in $B$ ) we obtain 17

$$
\alpha_{x y}^{\mathrm{SC}}=\frac{1}{6 \pi} \frac{e}{\hbar} \frac{\xi_{a b}^{2}}{\ell_{B}^{2} s} \frac{1}{\sqrt{1+\left(2 \xi_{c} / s\right)^{2}}} .
$$

Here, $\xi_{a b}\left(\xi_{c}\right)$ is the temperature-dependent coherence length parallel (perpendicular) to the layers, $s$ is the interlayer spacing, and $\ell_{B}$ the magnetic length. Note that the result essentially depends on one quantity, the coherence length, making this result particularly suitable for comparison with experiment.

In the Gaussian approximation, the coherence length diverges at $T_{c}^{\mathrm{MF}}, \xi_{a b / c}=\xi_{a b / c}(0) \sqrt{T_{c}^{\mathrm{MF}} /\left(T-T_{c}^{\mathrm{MF}}\right)}$. In reality, $\alpha_{x y}$ does not diverge at $T_{c}^{\mathrm{MF}}$ due to fluctuations beyond the Gaussian order which work to suppress $T_{c}$. The self-consistent Hartree approximation is perhaps the simplest way to go beyond the Gaussian approximation, and should work to some extent as the temperature is lowered below $T_{c}^{\mathrm{MF}}$. In this approximation, Eq. (2) remains valid, but the coherence lengths are renormalized from their Gaussian value to a lower value, diverging at $T_{c}$.

We digress, briefly, to mention that our Gaussian result also arises, with appropriate identification of parameters, as the Aslamazov-Larkin contribution in the microscopic theory of fluctuations about the BCS theory. In the same microscopic theory there are other diagrams, rather a lot of them really, that must be considered. It has been shown by one of us that all other contributions are subdominant near $T_{c}{ }^{37}$ For the cuprates the applicability of such microscopic computations is unclear which is why we prefer our current critical dynamics perspective. Finally, we also mention that the results of the Gaussian calculation may also be extended to finite magnetic field ${ }^{38}$

5. Overdoped $L S C O, T_{c}^{\mathrm{MF}}$, and $T_{\text {onset }}$ : Assuming the coherence lengths are known, the Gaussian approximation may be used to estimate $T_{c}^{\mathrm{MF}}$. Indeed, by using 


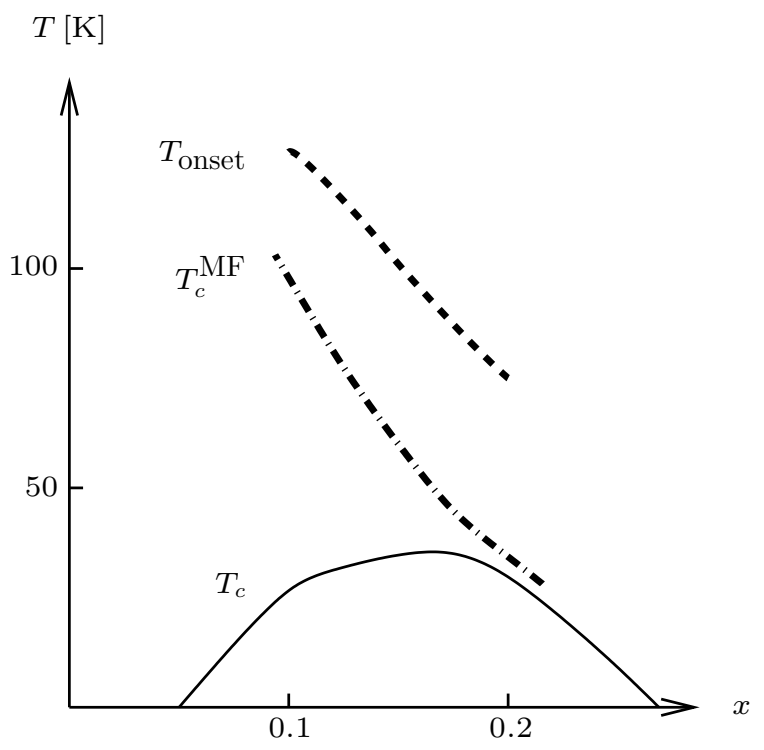

FIG. 2: Onset temperature of the Nernst effect, $T_{\text {onset }}$, and a suggestion of what the mean field critical temperature $T_{c}^{\mathrm{MF}}$ may look like in LSCO, as a function of doping. The onset data is based on the measurements of Wang et al. ${ }^{41}$

reasonable numbers for the coherence lengths in overdoped LSCO and assuming $T_{c}^{\mathrm{MF}} \approx T_{c}$, we found that the Gaussian approximation gives quantitatively good agreement with the measured signal 17 This is a clear indication that $T_{c}^{\mathrm{MF}}$ cannot be much higher than $T_{c}$ (if it was, Gaussian fluctuations would predict a Nernst signal at high temperatures which is larger than the observed signal). At the same time, the onset temperature $T_{\text {onset }}$ is much higher. The value of $T_{\text {onset }}$ is determined by the magnitude of the fluctuation signal and the ability of the experiment to detect it above the normal state background. In the present case the onset temperature is high for the same reasons the Nernst effect provides such a useful probe to superconducting fluctuations, namely that it represents a signature of superconductivity in a property which is very small in the normal state. In particular, we note that $T_{\text {onset }}$ occurs well inside the region of Gaussian fluctuations.

For overdoped LSCO we thus find that in most of the temperature range above $T_{c}$ the Nernst signal is a result of Gaussian fluctuations. Close to $T_{c}$ one has the region where phase fluctuations dominate over amplitude fluctuations. While the upper boundary of this region remains somewhat ambiguously defined, it is clear from this discussion that it cannot be taken to be $T_{\text {onset }}$. Rather, together with $T_{c}^{\mathrm{MF}}$, it will be much closer to $T_{c}$. Indeed, at $x=0.20$ we have $T_{c}=28 \mathrm{~K}, T_{\text {onset }} \approx 75 \mathrm{~K}$, and the comparison of the data with our calculations suggests a $T_{c}^{\mathrm{MF}}$ of about $35 \mathrm{~K}$ or lower ${ }^{39}$ To conclude, the region where phase fluctuations dominate is a small region in the vicinity of $T_{c}$ despite the large range of temperatures in which a Nernst signal is observed.

6. Underdoped LSCO: What happens when one consid- ers underdoped samples? As we have pointed out in our paper $\frac{17}{t}$ the observed signal is significantly larger than the result for Gaussian fluctuations if $T_{c}^{\mathrm{MF}} \approx T_{c}$ using reasonable parameters. This implies that a significant regime of non-Gaussian fluctuations must be included if one is to make sense of the experiment in terms of superconducting fluctuations. The interpretation is then that underdoping increases the strength of the fluctuations while increasing $T_{c}^{\mathrm{MF}}$, so that a systematically large gap between $T_{c}^{\mathrm{MF}}$ and $T_{c}$ is opened (see Fig. (2). As this happens, a large Nernst signal is expected from our analysis. We note that a similar doping dependence of $T_{c}^{\mathrm{MF}}$ and $T_{c}$ is at the heart of discussions of the pseudogap in terms of superconducting fluctuations; most notably in Emery and Kivelson's empirically deduced phase-crossover diagram for the cuprates ${ }^{12}$

There is however one more effect which may occur with underdoping, namely a change of the coherence length. The Nernst effect measurements at high fields performed by Wang et al ${ }^{40}$ suggest that the coherence length decreases as the sample becomes underdoped (however, to substantiate this result requires a theoretical understanding of the Nernst signal in this regime). Here, we note that decreasing the coherence length with underdoping has the effect of increasing both the range of magnetic fields in which fluctuation effects are observable (since $H_{c 2}$ becomes higher), and the effect of strong fluctuations (i.e., the separation between $T_{c}$ and $T_{c}^{\mathrm{MF}}$ ). These results are at the heart of the usual statement that a short coherence length leads to a large region for the observation of fluctuation phenomena in the cuprates. On the other hand, a shorter coherence length implies a weaker Gaussian contribution to $\alpha_{x y}$. On comparison with the usual implications of a short coherence, this result is quite counterintuitive! This suggests that as the sample goes underdoped, $T_{c}^{\mathrm{MF}}$ approaches $T_{\text {onset }}$ as the region of observability of Gaussian fluctuations decreases. One should remember, however, that the arguments regarding the position of $T_{c}^{\mathrm{MF}}$ in the underdoped regime are of the hand-waving type, and are not supported by a quantitative fit at this stage.

7. The "pseudogap": One of the main interests in the Nernst experiments arises from the possible implications for the physics of the pseudogap regime in the cuprates. Clearly, our analysis suggests that strong superconducting fluctuations exist in underdoped cuprates. One possibility, is to assume that all pseudogap phenomena arise somehow due to the same reason. Alternatively, it has been suggested that the pseudogap arises due to a spin gap, or one of several possible suggestions for a competing order. Or it could be that both piece of physics are operative in the pseudogap region. Here, we consider what can be said about this issue from the perspective of the Nernst effect.

The pseudogap region is observed in a wide variety of experimental probes (including NMR, various resistivity measurements, and heat capacity), roughly below a temperature scale $T^{*}, 10$ We therefore would like to ask if 
(and how) $T^{*}$ is related to $T_{c}^{\mathrm{MF}}$. The pseudogap temperature $T^{*}$ is itself a crossover temperature scale, so it is not well defined. Still, in experiments it is typically higher than $T_{\text {onset }}$ (in LSCO, it is roughly linear in doping, decreasing from about $350 \mathrm{~K}$ at $x=0.08$ to about $90 \mathrm{~K}$ at $x=0.22$, as derived from heat capacity measurements). In particular, it is sufficiently higher than $T_{c}^{\mathrm{MF}}$ to suggest that $T^{*}$ and $T_{c}^{\mathrm{MF}}$ are two distinct temperature scales. Of course, different measurements may yield different results for a crossover scale. On the other hand, because the Nernst effect is probably the most sensitive available probe to superconducting fluctuations, as an empirical matter one would expect that it yields a higher crossover scale arising from superconducting fluctuations than in other quantities. These observations suggest that $T^{*}$ is not set by superconducting fluctuations but is of a different physical origin. In particular, it may well be related to a spin gap or a competing order as suggested in the literature. However, the Nernst effect does suggest that superconducting fluctuations are an important part of the story at lower temperatures, and must be incorporated in the theoretical description of the pseudogap regime.

8. Other fluctuation phenomena: If the Nernst effect in the cuprates indeed arises from superconducting fluctuations, the question arises whether similar effects can be observed in other measurements. On the one hand, there are several measurements supporting the picture of strong fluctuations. These include ac conductivity ${ }^{42}$ and thermodynamic measurements $\stackrel{43}{\underline{3}}$ On the other hand, the dc conductivity and magnetization do not show an enhancement which can immediately be credited to strong superconducting fluctuations. It is therefore important to check whether these observations may be reconciled with our interpretation of the Nernst effect.

The contribution of superconducting fluctuations to the magnetization and conductivity (at the Gaussian level) are well known: ${ }^{44}$ For the magnetization, such contribution is expected to be very small and difficult to detect in the present case, except very close to $T_{c}$. With the conductivity the situation is different, i.e., the usual Aslamazov-Larkin result for the conductivity does not give a very small contribution already for Gaussian fluctuations. However, it must be noted that this result is invariably based on BCS microscopics, in particular, on the BCS value for the order parameter relaxation rate $\Gamma$. The fluctuation contribution to the conductivity is inversely proportional to $\Gamma$ (while the Nernst is independent of it). Hence, it is certainly possible to reconcile the absence of large fluctuation contributions to the conductivity in experiment with our interpretation if $\Gamma$ is substantially larger then its BCS value. At present there is no microscopic calculation supporting this; however, one possibility which comes to mind is that competing orders in the pseudogap phase provide a mechanism for a fast relaxation of the superconducting order parameter (see also Ref. 21).

In conclusion, in this paper we discussed the Nernst effect in the cuprate and its interpretation in terms of superconducting fluctuations. Our discussion is based on our previously published quantitative calculation,$\frac{17}{} \mathrm{Al}-$ ready at the level of Gaussian fluctuations our results find substantial superconducting fluctuations. Strong fluctuations, with a substantial separation between $T_{c}^{\mathrm{MF}}$ and $T_{c}$, occur in this region. On the other hand, $T_{c}^{\mathrm{MF}}$ is significantly lower than $T^{*}$, suggesting a different source for other pseudogap phenomena in the upper reaches of the pseudogap region.

We thank Steve Kivelson, Tom Lubensky, Subroto Mukerjee, Phuan Ong, Yayu Wang, and especially David Huse for enlightning conversations and their valuable input. This work was supported by the National Science Foundation through grants EIA-02-10736 and DMR-0213706 and by the David and Lucile Packard Foundation.
1 Z. A. Xu, N. P. Ong, Y. Wang, T. Kakeshita, and S. Uchida, Nature 406, 486 (2000).

2 Y. Wang, Z. A. Xu, T. Kakeshita, S. Uchida, S. Ono, Y. Ando, and N. P. Ong, Phys. Rev. B 64, 224519 (2001).

3 C. Capan, K. Behnia, J. Hinderer, A. G. M. Jansen, W. Lang, C. Marcenat, C. Marin, and J. Flouquet, Phys. Rev. Lett. 88, 056601 (2002); C. Capan, K. Behnia, Z. Z. Li, H. Raffy, and C. Marin, Phys. Rev. B 67 100507(R) (2003).

${ }^{4}$ H. H. Wen, Z. Y. Liu, Z. A. Xu, Z. Y. Weng, F. Zhou, and Z. X. Zhao, Europhys. Lett. 63, 583 (2003).

${ }^{5}$ H.-C. Ri, R. Gross, F. Gollnik, A. Beck, R. P. Huebener, P. Wagner, and H. Adrian, Phys. Rev. B 50, 3312 (1994); R. P. Huebener, Supercond. Sci. Technol. 8, 189 (1995) and references therein.

6 T. T. M. Palstra, B. Batlogg, L. F. Schneemeyer, and J. V. Waszczak, Phys. Rev. Lett. 64, 3090 (1990).

7 S. J. Hagen, C. J. Lobb, R. L. Greene, M. G. Forrester, and J. Talvacchio, Phys. Rev. B 42, 6777 (1990).

8 J. A. Clayhold, A. W. Linnen, Jr., F. Chen, and C. W.
Chu, Phys. Rev. B 50, 4252 (1994).

9 C. Hohn, M. Galffy, and A. Freimuth, Phys. Rev. B 50, 15875 (1994).

${ }^{10}$ For a review see T. Timusk and B. Statt, Rep. Prog. Phys. 62, 61 (1999).

11 M. Randeria, N. Trivedi, A. Moreo and R. T. Scalettar, Phys. Rev. Lett. 69, 2001 (1992); M. Randeria, in Models and Phenomenology for Conventional and HighTemperature Superconductors, edited by G. Iadonisi, J. R. Schrieffer, and M. L. Chiofalo (IOS Press, Amsterdam, 1998), p. 53 (also at cond-mat/9710223).

12 V. J. Emery and S. A. Kivelson, Nature 374, 434 (1995).

13 The notion that fluctuations are important in the physics of superconductivity at low dopings has appeared in many guises in the work of many people. While a proper accounting of its history would be more appropriate to a full-scale review of the topic, here we mention the early contributions of Anderson, who referred to the importance of phase fluctuations in Ref. 14, the Uemura plot ${ }^{15}$ which pointed 
to the relationship between the superfluid density and $T_{c}$ later formalized by Emery and Kivelson and the paper by Doniach and Inui. 16

14 P. W. Anderson, Science 235, 1196 (1987).

15 Y. J. Uemura et. al., Phys. Rev. Lett. 62, 2317 (1989).

16 S. Doniach and M. Inui, Phys. Rev. B 41, 6668 (1990).

17 I. Ussishkin, S. L. Sondhi, and D. A. Huse, Phys. Rev. Lett. 89, 287001 (2002).

18 S. Tan and K. Levin, Phys. Rev. B 69, 064510 (2004).

19 H. Kontani, Phys. Rev. Lett. 89, 237003 (2002).

20 S. Mukerjee and D. A. Huse, cond-mat/0307005 (unpublished).

21 C. Honerkamp and P. A. Lee, cond-mat/0309469 (unpublished).

${ }^{22}$ P. A. Lee, Physica C 317-318, 194 (1999).

23 S. A. Kivelson et al., Rev. Mod. Phys. 75, 1201 (2003).

24 S. Sachdev, Rev. Mod. Phys. 75, 913 (2003).

25 S. Chakravarty, R. B. Laughlin, D. K. Morr, and C. Nayak, Phys. Rev. B 63, 094503 (2001).

26 V. Oganesyan and I. Ussishkin, cond-mat/0312588 (unpublished).

27 We note that in this model there is also a dependence on the ultraviolet cutoff and the way that it is implemented.

28 P. C. Hohenberg and B. I. Halperin, Rev. Mod. Phys. 49, 435 (1977).

29 S. R. Shenoy, Phys. Rev. B 40, 5056 (1989).

${ }^{30}$ We are indulging in somewhat standard abuse of language here. Vortices generically involve amplitude fluctuations since the order parameter will be suppressed inside the core. So the increased amplitude fluctuations refer to variations of the amplitude beyond this "minimum".

31 The full curve was recently analyzed numerically by Muk- erjee and Huse at finite magnetic field,$^{20}$ their result agrees with the Gaussian computation at high temperatures, as expected.

32 See, e.g., R. P. Huebener, Magnetic flux structures in superconductors (Springer-Verlag, Berlin, 1979), and references therein.

33 C. Caroli and K. Maki, Phys. Rev. 164, 591 (1967).

34 R. J. Troy and A. T. Dorsey, Phys. Rev. B 47, 2715 (1993).

35 N. R. Cooper, B. I. Halperin, and I. M. Ruzin, Phys. Rev. B 55, 2344 (1997).

36 Indeed Mukerjee and Huse ${ }^{20}$ have given evidence that the entropy transported is configurational.

37 I. Ussishkin, Phys. Rev. B 68, 024517 (2003).

38 I. Ussishkin, S. L. Sondhi, D. A. Huse (unpublished).

39 There is also support to this picture from the numerical simulations of Mukerjee and Huse, ${ }^{20}$ who fit data for this sample below $T_{c}$ at finite magnetic field with twodimensional simulation results using $T_{c}^{\mathrm{MF}}=39 \mathrm{~K}$.

40 Y. Wang et al., Phys. Rev. Lett. 88, 257003 (2002); Y. Wang et al., Science 299, 86 (2003).

41 For $x \leq 0.07$ Wang et al. determine the onset temperature from $\alpha_{x y}$ instead of the Nernst signal, and obtain an onset temperature which rapidly decreases as the sample becomes more underdoped, and reaches zero at $x=0.03$. It is not clear what implications this result has on the behavior of $T_{c}^{\mathrm{MF}}$ in the very underdoped regime.

42 J. Corson et al., Nature 398, 221 (1999).

43 C. Meingast et al., Phys. Rev. Lett. 86, 1606 (2001).

44 For a recent review on superconducting fluctuations, see A. I. Larkin and A. A. Varlamov, in The physics of Superconductors, Vol. I, edited by K.-H. Bennemann and J. B. Ketterson (Springer, Berlin, 2003), p. 95. 